\title{
Emerging treatments in management of prostate cancer: biomarker validation and endpoints for immunotherapy clinical trial design
}

This article was published in the following Dove Press journal:

ImmunoTargets and Therapy

16 December 2013

Number of times this article has been viewed

\author{
Susan F Slovin \\ Genitourinary Oncology Service, \\ Sidney Kimmel Center for Prostate \\ and Urologic Cancers, Memorial \\ Sloan-Kettering Cancer Center, \\ New York, NY, USA
}

\begin{abstract}
The rapidly emerging field of immunotherapy and the development of novel immunologic agents that have been approved in melanoma and successfully studied in lung cancer, kidney cancer, and prostate cancer have mandated that there be uniformity in clinical trial analysis beyond conventional survival endpoints and imaging. This includes some measure of determining whether the immunologic target is hit and how the treatment has impacted on the immune system in toto. While melanoma is leading the field towards these ends, there is some doubt that not all of the recent successes with immune therapies, for example, checkpoint inhibitors, will be effective for every cancer, and that the toxicities may also be different depending on the malignancy. This review serves to elucidate the current issues facing clinical investigators who perform immunologic trials targeted at patients with prostate cancer and discusses the challenges in assessing the right immunologic endpoints to demonstrate biologic/ immunologic targeting leading to clinical benefit.
\end{abstract}

Keywords: sipuleucel-T, prostate-specific antigen, prostate cancer, biomarkers, monoclonal antibodies, vaccines, cellular therapy

\section{Introduction}

The clinical continuum of prostate cancer progression has successfully been interrupted by the advent of several newly approved agents, all of which have shown survival benefit and have unique mechanisms of action to modulate or diminish growth of prostate cancer. There has already been extensive discussion of novel agents in the literature, which has introduced two new androgen receptor-directed therapies, enzalutamide (Xtandi ${ }^{\mathrm{TM}}$; Astellas Pharma, Inc., Northbrook, IL, USA), ${ }^{1}$ and abiraterone (Zytiga ${ }^{\mathrm{TM}}$; Janssen Biotech, Inc., Horsham, PA, USA) ${ }^{2}$, a second-line post-docetaxel chemotherapy, cabazitaxel (Jevtana ${ }^{\mathrm{TM}}$; Sanofi-Aventis, Bridgewater, NJ, USA), ${ }^{3}$ a cellular product immunotherapy (sipuleucel-T, Provenge ${ }^{\mathrm{TM}}$; Dendreon, Seattle, WA, USA), ${ }^{4}$ and an alpha-emitting bone-targeting radiopharmaceutical, radium-223 (alpharadin, Xofigo $^{\mathrm{TM}}$; Bayer Corp, Shawnee Mission, KS, USA). ${ }^{5}$ The mechanistic variability of these drugs and their approval in the setting of castrate metastatic disease in the postdocetaxel setting has led to studies that have demonstrated benefit in earlier stages of the disease continuum, ${ }^{6}$ thereby encouraging physicians to investigate the role of these drugs in the earlier disease setting. This flexibility has also inspired investigators to study these drugs in unique combinations with agents that are outside their drug class. As such, studying these drugs in the premetastatic state, ie, either as neoadjuvant or biochemically relapsed, hormone-naïve clinical state has become more desirable. 
Immunologic approaches have significant potential for impacting on all clinical states of prostate cancer. They can be used at any time along the disease continuum. The rationale for immunologic therapy is also based on the fact that prostate cancer, unlike other solid tumors, has several biomarkers of disease, ie, the serum markers prostate-specific antigen (PSA) and acid phosphatase, and more recently, circulating tumor cells (CTCs). While the latter remain to be clinically validated in the setting of Phase III trials, they can provide a unique opportunity to study changes in the amplification or expression of the androgen receptor, in addition to correlating with changes in tumor biology when the patient is on specific drug(s). Several studies in breast, ${ }^{7}$ colon, ${ }^{8}$ and prostate ${ }^{9-11}$ cancer have shown a correlation of survival with number of CTCs in patients receiving cancer treatment. CTCs can be detected in as little as $7.5 \mathrm{cc}$ of peripheral blood per PAXgene ${ }^{\mathrm{TM}}$ (Qiagen, Venlo, Netherlands) tube. Patients with CTCs of five or more have been shown to have a poorer prognosis than those who have less than five. ${ }^{10,11}$ Similarly, in prostate cancer patients, for whom the standard biomarker (PSA) may be unreliable, a more reliable assessment of biologic response to treatment may be gleaned via CTC measurement, ie, a patient whose post-treatment CTC count declines and reaches zero will likely derive biologic and radiographic benefit from treatment. ${ }^{11}$ Another potentially relevant biomarker obtained from peripheral blood with relevance to prostate cancer is prostate-specific transcripts. A recent study by Danila et al ${ }^{12}$ used a validated reverse transcriptase polymerase chain reaction assay to detect prostate-specific RNA in whole blood from 97 men with castrate metastatic prostate cancer and compared it with routine CTC collection. The gene markers included KLK3, KLK2, HOXB13, GRHL2, and FOXA1, with the plan to validate these as prognostic factors for overall survival. ${ }^{12}$ These genes were selected based on their overexpression in metastatic prostate cancer. A correlation was seen between detectable transcripts and CTC count. The authors concluded that the reverse transcriptase polymerase chain reaction assay was prognostic for survival. In addition, it has the discriminatory power to separate patients based on their risk phenotypes compared with standard CTC technology. ${ }^{12}$ These observations need to be validated in larger patient cohorts and across all clinical states of the disease.

Prostate cancer also has a variety of cell surface antigens, ie, PSA, prostatic acid phosphatase, prostate stem cell antigen, ${ }^{13}$ six transmembrane epithelial antigens of the prostate, ${ }^{14}$ prostatespecific membrane antigen, ${ }^{15}$ Globo $\mathrm{H},{ }^{16} \mathrm{Tn},{ }^{17} \mathrm{GM} 2,{ }^{18}$ and MUC 1 and $2,{ }^{18}$ that are either overexpressed or underglycosylated, and represent targets to which immunologic therapies can be directed. More recently, monoclonal antibodies to checkpoint molecules, ie, anti-CTLA-4 (ipilimumab [Yervoy ${ }^{\mathrm{TM}}$; BristolMeyers Squibb, New York, NY, USA]) ${ }^{19}$ and anti-PD-1 (nivolumab [Bristol-Myers Squibb]), ${ }^{20}$ have demonstrated dramatic antitumor responses and survival benefits in melanoma, renal cell, and nonsmall cell lung cancers. Either drug alone or in combination has been shown to have a durable impact on the disease. Ipilimumab administered alone or in combination with radiation therapy has been evaluated in a Phase I/II trial in patients with castrate metastatic prostate cancer and deemed to be safe, with several patients demonstrating long-term remission. ${ }^{22}$ However, while the known associated side effects of colitis, rash, transaminitis, and hypophysitis have often been associated with the treatment response, they are reasonably well managed with steroids for the greater part, but can still significantly impact quality of life in patients who receive the drug.

While this is an exciting time for immunotherapy, and drugs such as ipilimumab and nivolumab are being explored alone, in combination, or with chemotherapy or novel biologic agents, immunotherapy trials in prostate cancer are ongoing. ${ }^{21,22}$ Van den Eertwegh et $\mathrm{al}^{23}$ performed an open-label, single-center, dose-escalation study of ipilimumab given concurrently with a fixed dose of GVAX ${ }^{\circledR}$ (Cell Genesys, San Francisco, CA, USA), a granulocyte-macrophage colonystimulating factor-transduced allogeneic prostate cancer cell vaccine, with the overall endpoint of safety. All patients received a priming dose of GVAX intradermally on day 1 with subsequent intradermal injections every 2 weeks for 6 months. The vaccinations were combined with escalating doses of ipilimumab $(0.3,1,3$, or $5 \mathrm{mg} / \mathrm{kg})$ every 4 weeks. While well tolerated, clinical activity was observed at the $3 \mathrm{mg} / \mathrm{kg}$ dose level. Grade 2 hypophysitis, grade 1 and 2 colitis, and grade 3 transaminitis were observed. $\mathrm{A} \geq 50 \%$ decline in PSA from baseline was recorded in $25 \%$ of patients, all of whom had received ipilimumab 3 or $5 \mathrm{mg} / \mathrm{kg} .{ }^{23} \mathrm{GVAX}$ had previously been extensively studied in the Phase I/II setting followed by the Phase III VITAL-1 (Vaccine Immunotherapy with Allogenic Prostate Cancer Cell Lines 1) trial in 621 asymptomatic patients with castrate metastatic prostate cancer randomized to either GVAX alone or GVAX plus docetaxel and prednisone. ${ }^{24}$ At a median follow-up of 17.1 months, the median survival time was similar in the two treatment arms, ie, 20.7 in the vaccine arm and 21.7 in the combination $\operatorname{arm}(P=0.78)$. A late but favorable treatment effect was suggested by the KaplanMeier survival curves crossing at approximately 21 months (91 weeks). This was consistent with previously observed patterns of responses to this immunotherapy. VITAL-1 was 
terminated based on the results of a futility analysis conducted at the company's request via the study's independent data monitoring committee indicating that the trial had less than a $30 \%$ chance of meeting its predefined primary endpoint of an improvement in overall survival.

Two Phase III trials in patients with castrate metastatic prostate cancer, one with ipilimumab given alone or with radiation following docetaxel, as well as in the prechemotherapy state without radiation, have been accrued. Preliminary results for the former have now been presented. ${ }^{25}$ Approximately 800 patients were randomized 1:1 to receive either $8 \mathrm{~Gy}$ radiation to a bone lesion before receiving either ipilimumab at $10 \mathrm{mg} / \mathrm{kg}$ or placebo every 3 weeks for a total of four treatments with a maintenance schedule of every 3 months for eligible patients. The endpoint was overall survival. Median progression-free survival also favored ipilimumab over placebo (hazards ratio 0.70; 95\% confidence interval $0.61-0.82$ ), as did PSA declines of $\geq 50 \%$ in evaluable patients ( $13.1 \%$ versus $5.3 \%$, respectively). ${ }^{25}$ A recent report by Cha et $\mathrm{al}^{26}$ evaluated the cell repertoire diversity using a multiplex polymerase chain reaction assay that amplified rearranged B-cell receptors and T-cell receptors in pretreatment and post-treatment peripheral blood mononuclear cells from 46 patients with metastatic castrateresistant prostate cancer or metastatic melanoma treated with anti-CTLA-4 monoclonal antibody. Of the 97 paired samples that were analyzed, 46 (47\%) had increases and 22 (23\%) had decreases in T-cell receptor diversity by more than two-fold. None of the untreated sample pairs displayed any significant changes in diversity. Although preliminary, the authors concluded that CTLA-4 blockade can impact on T-cell receptor diversity via changes in clonal phenotype over time. ${ }^{26}$

In an attempt to further enhance immunogenicity, novel constructs using recombinant pox virus vaccines have been used in several clinical trials. The current platform expresses multiple costimulatory molecules plus a tumor-associated antigen such as PSA, now known as PSA-TRICOM (PROSTVAC ${ }^{\circledR}-\mathrm{V} / \mathrm{F}$; Bavarian-Nordis, Denmark). ${ }^{27}$ The construct included transgenes encoding for PSA, as well as a costimulatory triad in B7.1 (the ligand for T-cell surface antigens CD28, CTLA-4, lymphocyte function-associated antigen 3 , and intercellular adhesion molecule-1). A multicenter, randomized Phase II trial of 125 patients was performed..$^{27} \mathrm{~A}$ vaccinia-based vector was for priming followed by six planned fowlpox-based vector boosts. Patients were allocated $(2: 1)$ to PROSTVAC-VF plus granulocyte-macrophage colony-stimulating factor or to empty control vectors plus saline injections with the endpoint of progression-free survival, which was the same between both groups. However, at 3 years post study, the PROSTVAC-VF patients had superior overall survival, with 25 (30\%) of 82 patients alive versus seven $(17 \%)$ of 40 controls. The median survival in the vaccine arm was 8.5 months (25.1 versus 16.6 months for controls). ${ }^{27}$ These promising results have led to an ongoing Phase III clinical trial. In an effort to further analyze prognostic features within the trial which may portend benefit and/or survival, Campbell et $\mathrm{al}^{28}$ studied 141 patients who were treated with PROSTVAC-VF in a series of Phase II trials. They found that prevaccination antibody levels to the blood group A trisaccharide (BG-Atri) were correlated with survival to a degree that reached statistical significance. Longterm survival was approximately doubled in subjects with high titers of anti-BG-Atri immunoglobulin $\mathrm{M}$ relative to subjects with little or no pre-existing immunoglobulin M for BG-Atri. This survival correlation was found to be highly specific to vaccine treatment and therefore may represent a new and effective biomarker by which to assess treatment benefit. ${ }^{28}$

Based on clinical trials in melanoma, progress has been made regarding how to evaluate immunotherapeutic trials. Patients with melanoma may often show "pseudoprogression" while on treatment. They may feel considerably better, but their tumor may enlarge on scans. Preclinical studies by Ribas et $\mathrm{al}^{29}$ demonstrated that this pseudogrowth of the cancer correlates with infiltration of the tumor by immune cell populations that will transiently appear. If another radiologic evaluation is performed after waiting several further weeks, the tumor will shrink and often remit. It should be noted that this appears to be largely seen in melanoma and has not been demonstrated in any of the prostate cancer trials to date.

While imaging plays a major role in determining treatment response, there is still a question as to how to best know if the immunologic "target" is hit by the therapy. A survival benefit may be demonstrated by a therapy, ${ }^{4}$ thereby suggesting impact, but survival without any demonstrable change in a biomarker or a scan may not be acceptable for a patient. This review discusses these issues and potential solutions, to going forward toward trying to establish a biomarker that can be used for clinical trials.

\section{Biomarker development}

By definition, a "biomarker" is a laboratory measurement that reflects the activity of a disease process. This is in contradistinction to a "surrogate marker", that is a "laboratory measurement used in therapeutic trials as a substitute for a clinically meaningful endpoint. It is a direct measure of how a patient feels, functions, or survives and is expected to predict the effect of the therapy ${ }^{30}$." The US Food and Drug Administration recognizes that basing an approval on the effect of a drug on an unvalidated surrogate 
introduces additional uncertainty into the approval process. ${ }^{24}$ To date, the successful investigation and use of CTCs and their association with survival has led the way towards studying different biomarkers for a variety of cancers..$^{6-11}$ Other attempts at biomarker evaluation and association with response have included checking for pretreatment and post-treatment levels of a known soluble antigen that may have been used as an immunogen or the expression of which is closely associated with disease, antibody titers, immune cell populations in serum, or assessment of tumor-infiltrating lymphocytes. ${ }^{31,32}$ Cancer is often thought of as a T-helper 2-dominant disease either with excess of interleukin (IL)-4, IL-5, IL-10, transforming growth factorbeta production with a therapeutically driven shift back towards the T-helper 1 profile. A T-cell signature that includes frequency and function of circulating T-cells via EliSpot, cytokine flow cytometry, tetramer binding can also be used. Apoptosis of CD8+ cells, a unique B-cell signature, suppressor cells in the microenvironment, which include regulatory T-cells/myeloid-derived suppressor cells (MDSCs) in human tumors, or observation of CD4+FOXP3+CD24 ${ }^{\text {high }}$ regulatory T-cells (often associated with a poor prognosis) all remain important avenues for immune evaluation. Cytokine gene or protein profiling are well suited to evaluation of the tumor microenvironment. There is also the potential to capture differences in patterns of their cytokine production that may be correlated with clinical response.

Concerns arise as to whether or not immune "responses" to cancer are really potential biomarkers of prognosis. Whiteside ${ }^{33}$ has pointed out that there have been numerous challenges trying to associate correlative immunologic parameters with clinical endpoints. She notes that expression of IgG kappa chain on tumor-infiltrating lymphocytes as well as B-cell and T-cell signatures have been validated as immunologic biomarkers. She postulates based on preclinical data that tumor-induced immune suppression exists and can promote tumor escape. As such, this premise could be further extrapolated in the pre and post treatment milieu by measuring changes in immune suppression using a variety of parameters, many of which are currently being validated. Another question that often arises is whether a panel of immune assays which can yield a signature that is applicable for every immune therapy or in fact for every disease as specific biomarkers may be needed for different diseases and different drugs.

\section{Biomarker assessment within the tissue environment}

The neoadjuvant setting can provide a direct way of interrogating the tumor and normal tissue microenvironment (Table 1). The benefit of testing an immune therapy either
Table I Milieux for immunologic interrogation

\begin{tabular}{ll}
\hline Neoadjuvant (early) & Metastatic (late) \\
\hline - Direct interrogation of tissue & - High tumor burden \\
and stroma & - Biology of disease different at met \\
- Impact on local disease to p0 & sites: bone vs LN vs visceral mets \\
or to MRV ( $<10 \%$ residual dz) & - Not every site of known disease \\
- Questionable impact on systemic & is active \\
$\begin{array}{l}\text { progression unless validated } \\
\text { in high risk population }\end{array}$ & - Immune cells can be crowded out \\
- Hard to design trials due & \\
to long natural history & \\
\hline
\end{tabular}

Abbreviations: vs, versus; LN, lymph node; MRV, mean residual viable tumor cells.

systemically or directly in a tumor or organ containing a tumor site lends itself to direct investigation. Not only can one determine the impact of a systemic or local therapy by evaluating the regression or remission of the cancer, but determination of tumor-infiltrating cell populations post versus pre therapy can also be performed. While the ease of this approach rests on the fact that pre and post treatment biopsies can be performed easily and the tissue appropriately interrogated, it remains unclear whether the treatment can impact on micrometastatic disease that could have escaped prior treatment. Nevertheless, this approach may offer some signal as to how a particular treatment may impact on a specific disease parameter. Precedence for this approach has been established in preclinical work by Krejci et al, ${ }^{34}$ and clinically in a small clinical trial of patients with bladder cancer ${ }^{35}$ undergoing neoadjuvant treatment with ipilimumab followed by a cystectomy trial that demonstrated an increased frequency of CD4+ICOShi T-cells, sustained over a period of 12 weeks of therapy and correlated with an increased likelihood of clinical benefit consisting of overall survival. The identification of ICOS as the first immunologic marker in both tumor tissues and in the peripheral blood of patients treated with anti-CTLA-4 may be of potential benefit as a marker of immune monitoring upon which other neoadjuvant trials may be built. Using a similar approach, a Phase II trial of the impact of neoadjuvant sipuleucel-T is ongoing in patients destined to undergo prostatectomy.

\section{Can drugs influence potential identification of a biomarker?}

A wealth of information regarding immunologic correlates has been gleaned through the experience with ipilimumab in melanoma, and new guidelines regarding evaluation of response parameters in melanoma have recently been established by the Society for Immunotherapy of Cancer. ${ }^{36}$ Despite the success in melanoma, one size does not fit all in terms of standardizing immune monitoring. True, there has 
been standardization and harmonization of immune assays, but the same criteria that subserve the analysis of a particular solid tumor may not hold true in another cancer. Therefore, a fair amount of circumspection is needed as to whether independent criteria that are specific for a particular cancer and/or a specific immune drug are important. To this end, recent work by a number of authors suggests that several parameters may potentially be considered to assess immune response to a particular drug. These include B-lymphocyte and T-lymphocyte profiling, assessment of absolute lymphocyte count (ALC), absolute and relative eosinophil counts pre and post treatment, expression of antigen and antigen ligand, and MDSCs.

\section{Ipilimumab and anti-PD- I as foundations for establishment of immunologic response criteria}

Multiple clinical trials in melanoma have highlighted the clinical value of ipilimumab and, for the first time, demonstrated an immune impact through a variety of different parameters. Many of these parameters fulfill a role as surrogate markers in that they are associated with a biological change in the disease as well as survival. More recently, the anti-PD-1 monoclonal antibody entered the clinical arena and was found to have unexpected efficacy. ${ }^{31,32}$ Programmed cell death protein 1 (PD-1) is a protein that in humans is encoded by the PDCD1 gene which encodes a cell surface membrane protein of the immunoglobulin superfamily. It has two ligands, PD-L1 and PD-L2, which are members of the B7 family. ${ }^{37,38}$ The PD-L1 protein is upregulated on macrophages and dendritic cells in response to treatment with lipopolysaccharide and granulocyte-macrophage colony-stimulating factor, and on T-cells and B-cells upon T-cell receptor and B-cell receptor signaling, whereas in resting mice, PD-1 is expressed on the surface of activated T-cells, B-cells, and macrophages, suggesting that, compared with CTLA-4, it can more broadly and negatively regulate immune responses. This has been confirmed in PD-1 knockout mice that have been shown to develop lupus-like glomerulonephritis and cardiomyopathy on C57BL/6 and BALB/c backgrounds, respectively. ${ }^{39,40}$ T-cells stimulated in vitro with anti-CD3 and treated subsequently with PD-1 ligand resulted in reduced T-cell proliferation and interferongamma production. ${ }^{30}$ Recent work by Topalian et $\mathrm{al}^{31}$ and Brahmer et $\mathrm{al}^{32}$ has demonstrated broader efficacy of antiPD-1 in several malignancies including melanoma, renal cell carcinoma, and nonsmall cell lung cancers. Unexpectedly, it was observed that overexpression of the PD-1 ligand could correlate with treatment response. In addition, this was the first time that concurrent clinical testing of antibodies blocking an immune regulatory receptor and one of its cognate ligands has been reported.

The availability of clinical material from patients enrolled in several clinical trials using ipilimumab alone and in combination with chemotherapy and biologics in melanoma has enriched efforts toward immunologic biomarker discovery and profiling compared with other solid tumors. Recent data reported by Schindler et $\mathrm{al}^{41}$ suggest that pretreatment levels of absolute and relative eosinophil counts are associated with improved overall survival in patients with metastatic melanoma treated with ipilimumab. A retrospective multicenter analysis was performed in 123 patients with stage III or IV melanoma who had received treatment with ipilimumab in the first-line and second-line setting at the approved dose of $3 \mathrm{mg} / \mathrm{kg}$ every 4 weeks. A baseline absolute eosinophil count of $\geq 0.1\left(10^{9} / \mathrm{L}\right)$ was associated with improved overall survival $(P=0.002)$, with significantly improved survival rates of $79 \%, 60 \%$, and $48 \%$ at 6,12 , and 18 months compared with rates of $48 \%, 37 \%$, and $19 \%$, respectively, for patients with a baseline eosinophil count below 1. Although retrospective, relative eosinophil counts also seemed to have similar benefits, suggesting the potential of these values as an immune-mediated response biomarker.

Another preliminary report by $\mathrm{Ku}$ et al, ${ }^{42}$ detailed a single-institution experience with ipilimumab used as a compassionate drug for patients with advanced melanoma. Patients with an ALC $>1,000 / \mu \mathrm{L}$ after two ipilimumab infusions (week 7) had a significantly improved clinical benefit rate $(P=0.01)$ and a median overall survival of 11.9 versus 1.4 months for patients with an ALC $<1,000 / \mu \mathrm{L}$. These observations served as the foundation for further studies by Postow et $\mathrm{al}^{43}$ who evaluated data from six studies of ipilimumab with or without dacarbazine, a standard chemotherapy used in melanoma. ALC was measured at baseline, prior to each dose during induction at the established times of weeks one, 4, 7, and 10, and at the end of induction at week 13. In all studies, the ALC increased significantly over time in patients who received ipilimumab irrespective of whether they were being treated with or without chemotherapy. A positive association between rate of ALC increase and overall survival was seen after two treatment doses. However, the increase in ALC was not specifically predictive of an overall survival benefit from ipilimumab based on analysis of the original ipilimumab trial with treatment arms using single-agent ipilimumab versus combination with the glycoprotein 100 vaccine. An overall survival benefit was observed for ipilimumab relative to the vaccine arm 
regardless of rate of change in ALC $(P=0.14)$. Therefore, these data suggest that ALC cannot be regarded as a reliable biomarker in disease management at this time.

Recent data presented by Wolchok et $\mathrm{al}^{44}$ have demonstrated improved efficacy using nivolumab (anti-PD-1) in combination with ipilimumab compared with using either drug alone, with a manageable safety profile. An analysis of single versus combined use of these agents was done by Callahan et al, ${ }^{45}$ who investigated the question of whether previously identified putative biomarkers for ipilimumab or nivolumab monotherapy are relevant in the combination setting. Interestingly, objective responses were seen in patients who were negative and positive for PD-L1 by immunohistochemistry. Evaluation of this two-drug combination did not document a rise in ALC relative to baseline, but there were phenotypic changes in peripheral blood T-cell subsets, including increased percentages of CD4 and CD8 expressing HLA-DR, ICOS, and/or Ki67. Low ALC ( $<1.0$ at week 6-7) did not preclude overall response, with three of 12 patients with low ALC seen to be responders. This is in contradistinction with prior reports of achievement of ALC $>1.0$ after 6 weeks of treatment with ipilimumab being associated with favorable clinical outcomes. Of note, another potential biomarker, MDSC levels, which were considered to be at a lower frequency before treatment, were associated with an improved overall response when compared with patients showing high MDSC levels. ${ }^{45}$ The results of this analysis suggest that the overall response was independent of PD-L1 status or ALC compared with either drug alone in this small subset of patients.

\section{MDSC as a potential biomarker}

Reference was made earlier to evaluation of MDSCs in a recent analysis of trials in melanoma. It is known that these cells can increase in both tumor tissue and blood in cancer patients, and that they correlate with a poor clinical outcome. There are pitfalls to using these cells, including their phenotypic and functional heterogeneity within the myeloid compartment. The fact that there are different subsets, leads to difficulty when comparing results, ie, subset of HLA-DR ${ }^{\text {neg }}$ Lin $^{\text {neg }}$ MDSC in peripheral blood has cells with monocytic and granulocytic features for which each can be divided: $\mathrm{CD} 33+, \mathrm{CD} 11 \mathrm{~b}+, \mathrm{CD} 15+$, and CD14+. Each is different with respect to its mechanism of suppression. Kitano et $\mathrm{a}^{46}$ evaluated MDSCs in peripheral blood as a biomarker of clinical outcome in a pilot correlative study of patients with stage IV melanoma treated with ipilimumab $10 \mathrm{mg} /$ $\mathrm{kg}$. A lower MDSC quantity before treatment predicted improved overall survival $(P=0.002)$, with a trend towards a clinical benefit at week 24 imaging. This effect appeared to be independent of pretreatment or week 7 ALC. A general trend of increasing MDSC numbers by week 24 compared with pretreatment baseline seemed to be associated with patients who did not appear to achieve clinical benefit. The authors concluded that there may be some predictive benefit of MDSCs as a biomarker, but this issue will need further retrospective and prospective validation.

Recent work by Reichenbach and Finn ${ }^{47}$ suggests that a better understanding of immune responsiveness to therapy involves potential instances of crosstalk, whereby context and cell type in signaling pathways are activated in an attempt to predict later effects on the immune response. They point out that signal transducers may have various upstream activators, ie, the IL-6 receptor, IL-21 receptor, and CD40 L, all signaling through STAT3. The role of STAT3 signaling in the differentiation of T-helper subsets has been well defined, confirming that signaling profiles can be generated to demonstrate the response to a vaccine by virtue of a $\mathrm{CD} 4^{+} \mathrm{T}$-cell activation "fingerprint" in vivo.

\section{Conclusion}

Immune biomarkers may need to be custom-tailored not only to the disease but also to its treatment. There is a need to develop rapid throughput for immune correlates such that real-time immune analyses can be performed. Despite the plethora of data presented in numerous clinical trials using melanoma as the most interesting immunologic model, there is still a need for better trial design that incorporates viable immunologic endpoints able to demonstrate that there is an impact on tumor biology as well as a correlation between disease improvement and changes in immune criteria. It is essential that all future trials incorporate acquisition of clinical specimens to address these questions.

\section{Disclosure}

The author reports no conflicts of interest in this work.

\section{References}

1. Scher HI, Fizazi K, Saad F, et al. Increased survival with enzalutamide in prostate cancer after chemotherapy. $N$ Engl J Med. 2012;367: 1187-1197.

2. Fizazi K, Scher HI, Molina A, et al. Abiraterone acetate for treatment of metastatic castration-resistant prostate cancer: final overall survival analysis of the COU-AA-301 randomised, double-blind, placebo-controlled phase 3 study. Lancet Oncol. 2012;13:983-992.

3. de Bono JS, Oudard S, Ozguroglu M, et al. Prednisone plus cabazitaxel or mitoxantrone for metastatic castration-resistant prostate cancer progressing after docetaxel treatment: a randomised open-label trial. Lancet Oncol. 2010;376:1147-1154. 
4. Kantoff PW, Higano CS, Shore ND, et al. Sipuleucel-T immunotherapy for castration-resistant prostate cancer. N Engl J Med. 2010;363:411-422.

5. Parker C, Nilsson S, Heinrich D, et al. Alpha emitter radium-223 and survival in metastatic prostate cancer. $N$ Engl J Med. 2013;369:213-223.

6. Ryan CJ, Smith MR, de Bono JS, et al. Abiraterone in metastatic prostate cancer without previous chemotherapy. N Engl J Med. 2013;368: 138-148.

7. Cristofanilli M, Budd GT, Ellis MJ, et al. Circulating tumor cells, disease progression, and survival in metastatic breast cancer. $N$ Engl $J$ Med. 2004;351:781-791.

8. Cohen SJ, Punt CJ, Iannotti N, et al. Relationship of circulating tumor cells to tumor response, progression-free survival, and overall survival in patients with metastatic colorectal cancer. J Clin Oncol. 2008;26: 3213-3221.

9. de Bono JS, Scher HI, Montgomery RB, et al. Circulating tumor cells predict survival benefit from treatment in metastatic castration-resistant prostate cancer. Clin Cancer Res. 2008;14:6302-6309.

10. Danila DC, Fleisher M, Scher HI. Circulating tumor cells as biomarkers in prostate cancer. Clin Cancer Res. 2011;17:3903-3912.

11. Danila DC, Morris MJ, de Bono JS, et al. Phase II multicenter study of abiraterone acetate plus prednisone therapy in patients with docetaxeltreated castration-resistant prostate cancer. J Clin Oncol. 2010;28: 1496-1501.

12. Danila DC, Anand A, Schultz N, et al. Analytic and clinical validation of a prostate cancer-enhanced messenger RNA detection assay in whole blood as a prognostic biomarker for survival. Eur Urol. July 26, 2013. [Epub ahead of print.]

13. Reiter RE, Gu Z, Watabe T, et al. Prostate stem cell antigen: a cell surface marker overexpressed in prostate cancer. Proc Nat Acad Sci U S A. 1998;95:1735-1740.

14. Danila DC, Szmulewitz RZ, Higano CS, et al. A phase I study of the safety and pharmacokinetics of DSTP3086S, an anti-STEAP1 antibodydrug conjugate (ADC), in patients (pts) with metastatic castrationresistant prostate cancer (CRPC). J Clin Oncol. 2013;31 Suppl 15S Abstr 5020.

15. Ghosh A, Heston WD. Tumor target prostate specific membrane antigen (PSMA) and its regulation in prostate cancer. $J$ Cell Biochem. 2004;91:528-539.

16. Slovin SF, Ragupathi G, Fernandez C, et al. A bivalent conjugate vaccine in the treatment of biochemically relapsed prostate cancer: a study of glycosylated MUC-2-KLH and Globo H-KLH conjugate vaccines given with the new semi-synthetic saponin immunological adjuvant GPI-0100 OR QS-21. Vaccine. 2005;23:3114-3122.

17. Slovin SF, Govindaswami R, Musselli C, et al. Fully synthetic carbohydrate-based vaccines in biochemically relapsed prostate cancer: clinical trial results with $\alpha-\mathrm{N}$-acetylgalactosamine-O-serine/threonine conjugate vaccine. J Clin Oncol. 2003;21:4292-4298.

18. Slovin SF. Emerging role of immunotherapy in the management of prostate cancer. Oncology. 2007;21:326-333.

19. Hodi FS, O'Day SJ, McDermott DF, et al. Improved survival with ipilimumab in patients with metastatic melanoma. $N$ Engl $J$ Med. 2010;363:711-723.

20. Wolchok JD, Kluger $\mathrm{H}$, Callahan MK, et al. Nivolumab plus ipilimumab in advanced melanoma. New Engl J Med 2013;369(2):122-133.

21. Madan RA, Mohebtash M, Arlen PM, et al. Ipilimumab and a poxviral vaccine targeting prostate-specific antigen in metastatic castrationresistant prostate cancer: a phase I dose-escalation trial. Lancet Oncol. 2012;13:501-508.

22. Slovin SF, Higano CS, Hamid O, et al. Ipilimumab alone or in combination with radiotherapy in metastatic castration-resistant prostate cancer: results from an open-label, multicenter phase I/II study. Ann Oncol. 2013;24:1813-1821.

23. van den Eertwegh AJ, Versluis J, van den Berg HP, et al. Combined immunotherapy with granulocyte-macrophage colony-stimulating factor-transduced allogeneic prostate cancer cells and ipilimumab in patients with metastatic castration-resistant prostate cancer: a phase 1 dose-escalation trial. Lancet Oncol. 2012;13:509-517.
24. Higano C, Saad F, Somer B, et al. A phase III trial of GVAX immunotherapy for prostate cancer versus docetaxel plus prednisone in asymptomatic, castration-resistant prostate cancer (CRPC). Genitourinary Cancer Symposium. J Clin Oncol. 2009;27 Suppl 15S: Abstr LBA150.

25. Gerritsen WR, Kwon ED, Fizazi K, et al. CA184-043: a randomized, multicenter, double-blind phase 3 trial comparing overall survival (OS) in patients (pts) with post-docetaxel castration resistant prostate cancer (CRPC) and bone metastases treated with ipilimumab (ipi) vs placebo (pbo), each following single dose radiotherapy (RT). Proc Eur Cancer Congress. 2013;Abstr 2850.

26. Cha E, Hou Y, Klinger M, et al. Effect of anti-CTLA-4 antibody treatment on T-cell repertoire evolution in treated cancer patients. $J$ Clin Oncol. 2013;31 Suppl 15S:Abstr 3020.

27. Kantoff PW, Schuetz TJ, Blumenstein BA, et al. Overall survival analysis of a phase II randomized controlled trial of a Poxviral-based PSA-targeted immunotherapy in metastatic castration-resistant prostate cancer. J Clin Oncol. 2010;28:1099-1105.

28. Campbell CT, Gulley JL, Oyelaran O, Hodge JW, Schlom J, Gildersleeve JC. Serum antibodies to blood group A predict survival on PROSTVAC-VF. Clin Cancer Res. 2013;19:1290-1299.

29. Ribas A, Chmielowski B, Glaspy JA. Do we need a different set of response assessment criteria for tumor immunotherapy? Clin Cancer Res. 2009;15:7116-7118.

30. Katz R. Biomarkers and surrogate markers: an FDA perspective. NeuroRx. 2004;1:189-195.

31. Topalian SL, Hodi FS, Brahmer JR, et al. Safety, activity, and immune correlates of anti-PD-1 antibody in cancer. $N$ Engl J Med. 2012;366: 2443-2254.

32. Brahmer JR, Tykodi SS, Chow LQM, et al. Safety and activity of anti-PD-L1 antibody in patients with advanced cancer. $N$ Engl $J$ Med. 2012;366:2455-2465.

33. Whiteside TL. Immune responses to cancer: are they potential biomarkers of prognosis? Front Oncol. 2013;3:1-8.

34. Krejci KG, Markiewicz MA, Kwon ED. Immunotherapy for urological malignancies. J Urol. 2004;171(2 Pt 1):870-876.

35. Carthon BC, Wolchok JD, Yuan J, et al. Preoperative CTLA-4 blockade: tolerability and immune monitoring in the setting of a presurgical clinical trial. Clin Cancer Res. 2010;16:2861-2871.

36. Kaufman HL, Kirkwood JM, Hodi, FS, et al. The Society for Immunotherapy of Cancer consensus statement on tumour immunotherapy for the treatment of cutaneous melanoma. Nat Rev Clin Oncol. May 15, 2013. [Epub ahead of print.]

37. Freeman GJ, Long AJ, Iwai Y, et al. Engagement of the PD-1 immunoinhibitory receptor by a novel B7 family member leads to negative regulation of lymphocyte activation. J Exp Med. 2000;192:1027-1034.

38. Latchman Y, Wood CR, Chernova T, et al. PD-L2 is a second ligand for PD-1 and inhibits T cell activation. Nat Immunol. 2001;2:261-268.

39. Nishimura H, Nose M, Hiai H, Minato N, Honjo T. Development of lupus-like autoimmune diseases by disruption of the PD-1 gene encoding an ITIM motif-carrying immunoreceptor. Immunity. 1999;11: $141-151$.

40. Nishimura H, Okazaki T, Tanaka Y, et al. Autoimmune dilated cardiomyopathy in PD-1 receptor-deficient mice. Science. 2001;291: 319-322.

41. Schindler K, Harmankaya K, Postow MA, et al. Pretreatment levels of absolute and relative eosinophil count to improve overall survival (OS) in patients with metastatic melanoma under treatment with ipilimumab, an anti CTLA-4 antibody. J Clin Oncol. 2013;31 Suppl 15S:Abstr 9024.

42. Ku GY, Yuan J, Page DB, et al. Single-institution experience with ipilimumab in advanced melanoma patients in the compassionate use setting: lymphocyte count after 2 doses correlates with survival. Cancer. 2010;116:1767-1775.

43. Postow MA, Chasalow SD, Yuan J, et al. Pharmacodynamic effect of ipilimumab on absolute lymphocyte count (ALC) and association with overall survival in patients with advanced melanoma. J Clin Oncol. 2013;31 Suppl 15S:Abstr 9052. 
44. Wolchok JD, Kluger HM, Callahan MK, et al. Safety and clinical efficacy of nivolumab (anti-PD-1, BMS-936558, ONO-4538) in combination with ipilimumab in patients (pts) with advanced melanoma (MEL). J Clin Oncol. 2013;31 Suppl 15S:Abstr 9012.

45. Callahan MK, Horak CE, Curran MA, et al. Peripheral and tumor immune correlates in patients with advanced melanoma treated with combination nivolumab (anti-PD-1, BMS-936558, ONO-4538) and ipilimumab. J Clin Oncol. 2013;31 Suppl 15S:Abstr 2003.
46. Kitano S, Postow MA, Cortez C, et al. Myeloid-derived suppressor cell quantity prior to treatment with ipilimumab at $10 \mathrm{mg} / \mathrm{kg}$ to predict for overall survival in patients with metastatic melanoma. J Clin Oncol. 2013;31 Suppl 15S:Abstr 2518.

47. Reichenbach DK, Finn OJ. Early in vivo signaling profiles in MUC1specific CD4+ T cells responding to two different MUC1-targeting vaccines in two different microenvironments. Oncoimmunology. 2013;2:e234291-e2342910

\section{Publish your work in this journal}

ImmunoTargets and Therapy is an international, peer-reviewed open access journal focusing on the immunological basis of diseases, potential targets for immune based therapy and treatment protocols employed to improve patient management. Basic immunology and physiology of the immune system in health, and disease will be also covered. In addition, the journal will focus on the impact of manage-

\section{Dovepress}

ment programs and new therapeutic agents and protocols on patient perspectives such as quality of life, adherence and satisfaction. The manuscript management system is completely online and includes a very quick and fair peer-review system, which is all easy to use. Visit http://www.dovepress.com/testimonials.php to read real quotes from published authors.

Submit your manuscript here: http://www.dovepress.com/immunotargets-and-therapy-journal 